\title{
OP-0002-Recently diagnosed type 2 diabetes is associated with low pancreas volume and high intrapancreatic fat content
}

\author{
A Al-Mrabeh ${ }^{1}$, SV Zhyzhneuskaya ${ }^{1}$, C Peters $^{1}$, AC Barnes $^{2}$, KG Hollingsworth ${ }^{1}$, H Pilkington ${ }^{3}$, \\ MEJ Lean ${ }^{4}$, R Taylor ${ }^{1}$ \\ ${ }^{1}$ Magnetic Resonance Centre, Institute of Cellular Medicine, Newcastle University, Newcastle upon Tyne, UK \\ ${ }^{2}$ Human Nutrition Research Centre, Institute of Health and Society, Newcastle University, Newcastle upon Tyne, UK \\ ${ }^{3}$ Clinical Ageing Research Unit, Campus for Ageing and Vitality, Newcastle University, Newcastle upon Tyne, UK \\ ${ }^{4}$ School of Medicine, Dentistry and Nursing, University of Glasgow, Royal Infirmary Campus, Glasgow, UK
}

\section{Background}

Although type 2 diabetes (T2DM) does not develop without a major decrease in insulin secretion, the pancreas itself has been little studied. Previous work has shown that pancreas volume and fat content differ from normal in type 2 diabetes as quantified by precise magnetic resonance methods [1-3]. Studies to date have been relatively small, and the morphology of the pancreas in the early years after diagnosis of type 2 diabetes has not been established. In this study, the Tyneside baseline dataset of the prospective, randomised Diabetes Remission Clinical Trial (DiRECT) was examined [4].

\section{Methods}

Participants in DiRECT with complete metabolic and imaging data were studied ( $\mathrm{n}=89 ; 43 \%$ female; $53.1 \pm 7.8$ years; weight $99.5 \pm 16.2 \mathrm{~kg}$; BMI $\left.34.3 \pm 4.2 \mathrm{~kg} / \mathrm{m}^{2}\right)$. The mean duration of type 2 diabetes was $2.9 \pm 1.7$ years. Fat measurement using the 3-point Dixon with MR-opsy analysis and morphometric analysis were carried out using 3.0 Tesla Philips Achieva scanner [5,6]. Metabolic studies included fasting plasma insulin and glucose, and very low density lipoprotein (VLDL1) production measured by a non-isotopic competitive blocking method. For comparison, data from a normal control group was included.

\section{Results and Discussion}

Pancreatic fat content was raised at $8.3 \pm 2.3 \%$ (non-diabetic $6.2 \pm 2.2 \%$; $\mathrm{p}=0.0001$ ). The mean volume of the pancreas was low at $62.5 \pm 16.5 \mathrm{~cm}^{3}$ (normal $\left.79.8 \pm 14.4 \mathrm{~cm}^{3} ; \mathrm{p}<0.0001\right)$. Liver fat content was also elevated at $14.7 \pm 9.8 \%$, and VLDL1-triglyceride production rate was $545 \pm 179 \mathrm{mg} / \mathrm{kg} /$ day leading to a total VLDL1-triglyceride pool size of $2291 \pm 1645 \mathrm{mg}$. Fasting plasma glucose and insulin were $8.4 \pm 2.6 \mathrm{mmol} / \mathrm{l}$ and 91.6 (24.1-313.0) pmol/1, respectively. Pancreas fat content correlated positively with BMI $(r=0.27, p=0.01)$ but not with VLDL production. Pancreas volume correlated with fasting plasma insulin $(\mathrm{r}=0.24, \mathrm{p}=0.02)$. Pancreas volume decreased with age $(\mathrm{r}=-0.25, \mathrm{p}=0.02)$ but was not related to diabetes duration within the narrow range of diabetes duration in this cohort. We reported previously [6] a strong inverse relationship between pancreas fat and volume, and this relationship is present even in this cohort with diabetes duration $<6$ years.

\section{Conclusions}

$\checkmark$ Low pancreas volume and high fat content of the pancreas are distinct characteristics of early type 2 diabetes.

$\checkmark$ Further work is required to determine whether a small pancreas predisposes to type 2 diabetes or whether this is a consequence of the disease process.

$\checkmark$ Pancreas volume and fat content may be useful as prognostic markers for progression of type 2 diabetes.
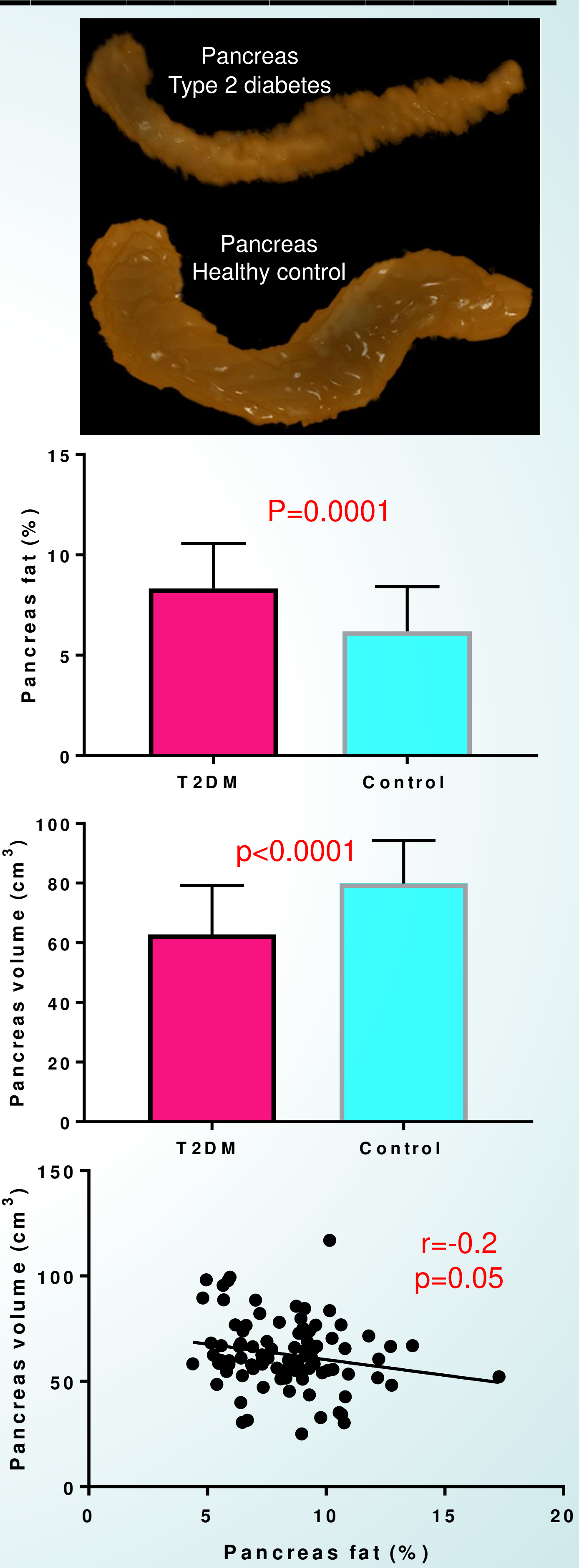

All data are presented as value $\pm \mathrm{SD}$

\section{References}

1-Lim et al. (2011) Diabetologia 54: 2506-2514. 2-Macauley et al. (2015). PLOS One 10: e0126825. 3-Steven et al. (2016). Diabetes care 39: 158-165. 4- Leslie et al. (2016). BMC Family Practice, 17, 20. 5-Al-Mrabeh et al. (2017) .PLOS One, 12(4), 1-19. 6-Al-Mrabeh et al. (2016). Diabetologia 59(8), 1753-1759 